\title{
Des-pisando las pruebas
}

\section{Taking Distance from the pisa tests}

\author{
Alix María Casadiego \\ Magíster en Educación. Universidad Surcolombiana. \\ Neiva. Colombia. \\ alixcasadiego@yahoo.es \\ Álvaro E. Avendaño R. \\ Magíster en Educación. Universidad Surcolombiana. \\ Neiva. Colombia. \\ alave@usco.edu.co
}

\section{Resumen}

A raíz de los resultados de los estudiantes colombianos en las pruebas pisa, los autores hacen un paralelo entre la educación colombiana y los aspectos legales, históricos, estructurales, de principios orientativos y curriculares que caracterizan la educación en cuba, paradigma educativo en América latina.

Palabras claves: Educación, formación, enseñanza, Cuba, Colombia, calidad, currículo, docente, aspectos legales, integral, estructura, legislación.

\section{Abstract}

Taking into account the results of Colombian students' results in the PISA test, the authors draw a parallel between Colombian education and the legal, historic, structural aspects and guiding and educational principles which characterize education in Cuba, the educational paradigm in Latin America.

Keywords: Education, training, teaching, Cuba, Colombia, quality, curriculum, teacher, legal aspects, integral, structure, legislation.

\section{Colombia en Pisa}

En Diciembre pasado, se conocieron los resultados de PISA en la prueba de mayo del 2012. Nueve mil estudiantes colombianos compitieron con $85 \mathrm{mil}$ estudiantes de 15 años de otros países en las pruebas de la Organización para la Cooperación y el Desarrollo Económicos (OCDE) del Programa Internacional de Evaluación de Estudiantes (PISA, por su sigla en inglés), que evalúa si los jóvenes están adquiriendo la habilidad necesaria para solventar situaciones problemáticas en el siglo XX. Colombia quedó en el último lugar.

Entre los aspectos para remedia estos resultados que los investigadores contratados por la Fundación Compartir proponen es aumentar la remuneración salarial y las bonificaciones, que alcanzaría en el 2030 para todos los docentes del país casi 2 billones de pesos del 2013 (Fundación Compartir, 2014). Mejores salarios atraería a mejores bachilleres o universitarios a esta profesión docente. Además de ofrecerles becas y ayudas económicas. Con lo que mermaría el impacto de la deplorable formación de los maestros.

La inyección de presupuesto debe estar aislada de la corrupción, la cual, según el propio MEN encontró 561.000 estudiantes fantasma en el país, o sea que las secretarías de educación regionales esquilman más de 850.000 millones de pesos al año. La mayoría de las secretarías son 'cuotas políticas' sin formación ni conocimiento de las prioridades en materia de inversión del sector (Revista Semana, 2014).

Un buen número de buenos maestros (masa crítica), que son los que influyen más que cualquier otro insumo escolar, haría innecesaria los sistemas continuos de evaluación que proponen algunos autores, acorde con la exitosa experiencia de Finlandia (Unesco, 2006), donde no hay evaluación externa, sólo una remuneración alta. Su mayor preocupación es 
atraer a los mejores candidatos hacia la profesión docente y mantenerlos motivados hasta su jubilación. Basta con mencionar que sólo 29 de los 57 países participantes en PISA, tienen establecidos exámenes nacionales de evaluación, como es el caso de la Prueba Saber (MEN, 2008), pero estas evaluaciones, a Colombia no le han significado calidad, como lo muestran los resultados PISA.

Desde los tiempos de Santander, quien creó escuelas y universidades cargo del estado, pasando por López Pumarejo, fundador en los años 30 de la Escuela Normal Superior para elevar la calidad de los maestros, por Alberto Lleras y el plebiscito de 1957 que aprobó mínimo el 10 por ciento del PIB para educación, hasta César Gaviria, con su Misión de Sabios en 1994, prácticamente todos los gobiernos desde la Independencia han proclamado la importancia de la educación, pero ninguno realmente cumplido. Para este año 2014 tenemos un 17,6\% del total del presupuesto (presidencia de la república, 2014) para educación, es decir, apenas cercano a un 3\% del PIB, según la publicación del Banco de la República sobre el PIB 2013 (Banco de la República, 2013) en la antesala de las pruebas Pisa próxima a realizarse en 2015 , que será en ciencias (Icfes, 2014).

\section{Legislación Vigente}

Cuba no tiene una ley especial para educación. La ley o norma que la rige es la misma constitución de 1976, que sus artículos $9,39,40,51$ y 52 , explicita los Fundamentos Políticos, Sociales y Económicos del Estado y declara que "no haya niño que no tenga escuela, alimentación y vestido.., que no tenga oportunidad de estudiar; que no tenga acceso al estudio, la cultura y el deporte...".

La base de la legislación Cubana está contenida en las Resoluciones $\mathrm{N}^{\mathrm{O}}$. 210 de 23 de abril de 1975 , No 654 de 21 de octubre de 1976, $\mathrm{N}^{\circ} 656$ de 21 de octubre de 1976 , No 160 de 17 de marzo de 1981 y No 430 de 24 de julio de 1981, del perfeccionamiento de la educación, dictadas por el Ministro de Educación,

La enseñanza es función del Estado y es gratuita. Se debe promover la educación patriótica y la formación de las nuevas generaciones y la preparación de los niños, jóvenes y adultos para la vida social, combinando la educación general y las especializadas con el trabajo, la investigación para el desarrollo, la educación física, el deporte y la participación en actividades políticas, sociales y de preparación militar.

\section{Evolución de la Educación}

El inicio de la enseñanza en la isla se debe a la labor de los frailes dominicos y franciscanos, alrededor de 1522. Alrededor de 1722 se creó en Santiago de Cuba el Seminario Conciliar de San Basilio Magno para enseñar Teología, Moral, Filosofía y Derecho Canónigo (Sistema Educativo Nacional de Cuba, 1995)
La enseñanza primaria se mantuvo precariamente a través de las escuelas públicas sostenidas con los escasos medios de la Sociedad Económica de Amigos del País y algunas escuelas privadas sostenidas por instituciones religiosas y maestros laicos.

Por Real Orden del 29 de diciembre de 1841 se exigió que la instrucción fuese gratuita para los niños verdaderamente pobres. En 1863 se establece la enseñanza obligatoria de los 6 a los 9 años de edad.

Hacia el año 1950 llama la atención que a pesar de ser Cuba un país agrícola, la enseñanza agropecuaria presentaba una situación crítica, pues sólo contaba con seis granjas escuelas y un instituto forestal con escasas posibilidades de matrícula y de recursos. El mayor nivel de desarrollo se apreciaba en las escuelas de comercio con los estudios de economía y administración. Existían 11 centros estatales y un número mayor controlado por organizaciones privadas.

A partir de Enero de 1959 con el triunfo de la Revolución se inician ingentes esfuerzos que tuvieron como contenido esencial dar solución a los grandes problemas del pasado neocolonial, la reorganización y tecnificación del Ministerio de Educación y la toma de medidas inmediatas para eliminar el analfabetismo y garantizar la extensión de los servicios educacionales.

Se dictó la Ley 561 del 15 de septiembre de 1959, que permitió la creación de 10.000 aulas de enseñanza primaria y la Ley 680 del 23 de diciembre de 1959, la cual estableció las normas para la reforma integral de la enseñanza. Ella define el Sistema Nacional de Educación, sus objetivos y niveles, los tipos de centros docentes y diferentes aspectos de la docencia, fija la obligatoriedad de la enseñanza hasta los 12 años y el $6^{\circ}$ grado, crea la escuela secundaria básica y establece el preuniversitario como institución de educación general media, previa a los estudios universitarios, entre otros aspectos.

En 1960 se constituyó el Contingente de Maestros Voluntarios, el cual estuvo formado por 3.000 jóvenes que marcharon a todos los rincones del país para llevar la educación. Posteriormente se constituyó la brigada de maestros de vanguardia "Frank París", la cual desarrolló su trabajo en las montañas de Oriente, Las Villas y en la Sierra de los Órganos en Pinar del Río. Mediante la campaña se redujo el analfabetismo de un 23,6 por ciento a un 3,9 por ciento, índice que ha continuado disminuyendo.

Desde Mayo de 1961 se habían dado los pasos iníciales para la formación de instructores de arte. Inspirada en el trabajo de los maestros voluntarios y brigadistas alfabetizadores que trabajan para llevar la cultura hasta las fábricas, granjas del pueblo, cooperativas de producción agrícola y unidades militares.

Para la formación regular de maestros primarios se estableció cursar un año de estudios en las montañas de las provincias de Oriente, a fin de que fueran capa- 
ces de adaptarse a las situaciones que pudieran encontrar posteriormente en el ejercicio de su profesión.

En ese mismo año de 1962 se iniciaron las experiencias de la vinculación de los estudiantes de nivel medio con los planes de desarrollo agropecuario. Este trabajo experimental se ha sistematizado; el principio de combinación del estudio con el trabajo es fundamental en la pedagogía cubana.

A partir de 1975, año en que comenzó la aplicación del Plan de Perfeccionamiento del Sistema Nacional de Educación, se han publicado más de 250 millones de ejemplares en libros, cuadernos de trabajo, orientaciones metodológicas y otros.

\section{Estructura del Sistema Educativo ${ }^{1}$}

Los subsistemas que integran la estructura del Sistema Nacional de Educación son:

- Educación Preescolar

- Educación General Politécnica y Laboral

- Educación Especial

- Educación Técnica y Profesional

- Formación y Perfeccionamiento del Personal Pedagógico

- Educación de Adultos

- Educación Superior

En Cuba el niño que ingresa a la Educación Primaria ha recibido atención educativa desde la edad preescolar, ya sea por la por vía institucional (círculo infantil) como por la no institucional (Programa "Educa a Tu Hijo"), lo que favorece su desarrollo.

La preescolar es obligatoria y atiende a los niños desde los 6 meses de nacido hasta los 5 años de edad y consta de tres componentes principales:

- El Círculo Infantil, institución preescolar para la atención y educación de los niños desde los 6 meses hasta los 5 años de edad.

- El grado preescolar, o grado preparatorio para la escuela para los niños de 5 años, y que puede estar indistintamente ubicado en un círculo infantil o una escuela primaria.

- Las vías no formales de la educación preescolar, para los niños que no asisten a las instituciones que propicie educación, mediante el concurso de los padres y la comunidad.

La Educación Primaria está estructurada en seis grados, agrupados en dos ciclos: uno de primero a cuarto grado y otro que incluye el quinto y sexto grados.

La educación en Cuba es obligatoria hasta el noveno grado de la enseñanza secundaria y gratuita en todos los niveles, incluyendo la superior.
El fin de la Educación Primaria es contribuir a la formación integral del escolar, fomentando desde los primeros grados, la interiorización de los conocimientos y orientaciones valorativas, que se reflejan gradualmente en los sentimientos, formas de pensar y comportamiento, que se corresponden con los valores e ideales de la Revolución Socialista.

Educación primaria: Los niños comienzan la escuela primaria a la edad de seis años. La educación primaria dura seis años (del 1ro al 6to grado).

Educación media: Se ofrece de 7mo a 9no grado, dura tres años y se desarrolla en la Escuela Secundaria Básica. También es posible estudiar en una escuelataller para adiestrarse en un oficio. Los egresados de la escuela secundaria obtienen un Diploma de Estudios secundarios terminados. Escuelas Tecnológicas: Ofrecen una educación general junto a un adiestramiento técnico, su terminación, con Diploma de Técnico Medio equivale a nivel medio terminado.

Educación Preuniversitaria o Media Superior: Terminada la Escuela secundaria los estudiantes pueden matricularse en los Institutos Preuniversitarios, con un ciclo de duración de tres años $(10 \mathrm{mo}$, 11vo y 12vo grado) como preparación y nivel precedente al nivel universitario. Al término se otorga un título de Bachiller. Culminan el nivel medio superior. Existen también las Escuelas de Formación Técnica Profesional, y vocacional que forman técnicos mediosuperior, conocidas también como Institutos Politécnicos, en ciclos de duración de tres o cuatro años de estudios. Al término reciben un Diploma de graduados en la especialidad técnica correspondiente que equivale al nivel medio superior y le permite continuar estudios superiores. La Educación Primaria es la base de la educación básica y tiene carácter obligatorio y universal, con beneficio para todos los niños y niñas, partiendo del principio de que a la más joven generación hay que enseñarla, atenderla y educarla. Comprende el grupo de edades entre 6 y 11 años.

\section{Educación Superior:}

\section{Estructura de los estudios universitarios.}

Las carreras universitarias en Cuba tienen un tiempo mínimo de duración de cinco cursos académicos. El curso académico se divide en períodos que pueden ser semestres, trimestres o bloques (estos últimos de dos meses de duración aproximadamente) muy usados para actividades laborales investigativas de los estudiantes.

Para ingresar a la educación superior, el estudiante debe haber vencido doce o trece grados de estudios precedentes, divididos en: a) Nivel primario (seis grados) b) Nivel secundario (tres grados) c) Nivel preuniversitario o técnico medio superior (tres y cuatro grados respectivamente).

1 - Fuente: Ministerio de Educación, Ministerio de Educación Superior y Comité Estatal de Estadística. 
En la actualidad en Cuba se ofrecen certificaciones de 95 títulos de conclusión de estudios de nivel superior o de pregrado. De ellos, el 30,5 \% (29) corresponde a República de Cuba Estructura y titulaciones de Educación Superior 15 las Ciencias Pedagógicas; el 17,8 \% (17) a las Ciencias Sociales y Humanísticas; otro 17,8\% (también 17) a las Ciencias Técnicas; el 13,6\%(13) a las Ciencias Naturales y Matemáticas; el 5,2 \% (5) a las Ciencias Agropecuarias; otro 5,2 \% (también 5) a las Ciencias Médicas; junto a otro 5,2\% (5) a las Artes; el $3,1 \%$ (3) a las Ciencias Económicas; y el 1 \% (1) a la Cultura Física y el Deporte.

El estudio de carreras de nivel superior o de pregrado en Cuba se organiza principalmente en tres tipos de cursos: los cursos regulares diurnos (CRD), con dedicación a tiempo completo, a los que acceden principalmente los jóvenes que culminan el nivel medio superior (preuniversitario); los cursos regulares para trabajadores (CPT), con dedicación a tiempo parcial, que se desarrollan para trabajadores que mantienen su vínculo laboral mientras estudian; y los cursos a distancia (EAD), en los cuales el régimen de estudio no es presencial, y donde el estudiante recibe apoyo mediante guías y textos, además de consultas establecidas con profesores, estando obligados a presentarse solamente a realizar los exámenes exigidos en el plan de estudio.

En la actualidad un nuevo modelo pedagógico se experimenta, dirigido a garantizar y estimular la continuidad de estudios de nivel superior, en gran parte asociado al proceso de universalización de la educación superior en el país, donde el estudiante recibe algunas horas de orientación del profesor, tutoría sistemática guías y materiales audiovisuales con un soporte importante en las tecnologías de la información y las comunicaciones, lo que otorga una mayor responsabilidad al estudiante en su actividad de estudio. Asimismo, asume una mayor flexibilidad en la organización del proceso y la culminación de estudios, para el cual se introducen nuevas normativas metodológicas y de reglamentación.

Entre los logros alcanzados se puede enunciar: la erradicación del analfabetismo, ha desaparecido el atraso escolar, las tasas de repitencia y abandono son bajas, la escolaridad media de la población es de $9^{\circ}$ grado, el país dispone de un docente por cada 45 habitantes, en la educación primaria todos los maestros poseen el título idóneo para ejercer y en el nivel medio el 87\% de los profesores son licenciados en educación, se han graduado en la educación técnica y profesional con nivel medio superior, cerca de un 1.400.000 estudiantes, más de treinta mil especialistas trabajan en instituciones científicas, no obstante, aún falta mucho por hacer para continuar elevando la calidad de la educación.

\section{Desarrollo Curricular en Cuba}

La concepción curricular de la educación superior satisface las siguientes ideas rectoras:
* Lograr una preparación básica para todos los alumnos pertenecientes a la Educación Preuniversitaria, independientemente del tipo de centro, durante los dos primeros años de su formación, (10mo y 11avo grados), asumiendo los mismos programas de estudio, software y video clases en las diferentes asignaturas.

* En el último año (12mo grado) pasan a un proceso de diversificación que constituye una especialización en cuatro ramas, según los siguientes grupos de carreras universitarias por las que opta el alumno:

- Ciencias Médicas, Agropecuarias, Biológicas y Cultura Física.

- Ciencias Técnicas, Naturales y Matemática.

- Ciencias Sociales, Humanísticas y Económicas.

- Ciencias Pedagógicas

El objetivo de la Educación Preuniversitaria es la formación integral de los jóvenes a partir del desarrollo de una cultura general, política y preprofesional sustentada en el principio martiano de estudiotrabajo, que garantice la participación protagónica e incondicional en la construcción y defensa del proyecto socialista cubano, y en la elección consciente de la continuidad de estudios superiores.

En el primer ciclo se imparten conocimientos esenciales de las materias instrumentales, Lengua Española y Matemática Además, nociones elementales relacionadas con la naturaleza y la sociedad. Se realizan actividades de educación física, laboral y estética, que contribuyen a la formación multilateral del educando, y en segundo ciclo continúa el desarrollo de las habilidades iniciadas en el primero y comienza el estudio de nuevas asignaturas como la Historia y la Geografía de Cuba, Ciencias Naturales y Educación Cívica. Además, se fortalecen las actividades de educación patriótica, física, laboral y estética, que contribuyen a la formación integral de los alumnos.

Como parte del currículo escolar se contempla y considera la educación extraescolar que complementa, refuerza y enriquece la labor docente-educativa de la escuela mediante actividades artístico-culturales, deportivas, recreativas, patrióticas, de formación vocacional y orientación profesional, así como en los concursos de conocimientos, competencias de habilidades y movimiento de monitores.

El curso se divide en cuatro períodos de clase, con una semana de receso docente entre uno y otro y una duración de $\mathbf{4 0}$ semanas en general, con un total de $\mathbf{1 0 0 0}$ horas clase.

\section{Concepción de Educación y de Currículo}

La educación en cuba es un proceso eminentemente investigativo ya que es un sistema de mejoramiento continuo, lo cual muestra que el proceso curricular, como es de esperarse, es una acción eminentemente 
investigativa y no procedimental e instrumental.

Tienen una metodología denominada Entrenamiento Metodológico Conjunto, mediante el cual, los profesores comparten sus experiencias y se ayudan unos a otros logrando así una mayor calidad en su trabajo.

El método de Entrenamiento Metodológico Conjunto (EMC), implementado con gran éxito en Cuba y ahora en Colombia (MEN, 2005) se orienta a lograr mayor calidad en el trabajo de los docentes y de otro personal que dirige diferentes instancias de la educación. Las fases del EMC comprenden el diagnóstico, la demostración, el control y la evaluación. Para tal fin, se diseñan clases en las que se precisan los estándares básicos, los logros obtenidos y esperados, así como las acciones didácticas, pedagógicas y metodológicas a seguir para tratar los contenidos del curso en cada área y se realizan clases demostrativas y abiertas, reuniones, talleres, seminarios y mesas redondas, entre otras actividades, que generan una dinámica de colaboración entre los maestros de una misma área. Es decir, la esencia del método es producir una nueva relación de trabajo entre los maestros, los directivos docentes encargados de acompañar a los docentes en el mejoramiento de sus clases y los funcionarios de las entidades territoriales.

Este programa se adelantó en Colombia con el apoyo de un grupo de expertos cubanos que entrenaron a 3.071 maestros y directivos docentes del país, quienes pueden replicar esta metodología en las demás escuelas de su jurisdicción (MEN, 2009).

\section{Principios que orientan el Sistema Educativo}

EL sistema nacional de educación es un proceso docente educativo integral, sistemático, participativo y en constante desarrollo, que se apoya en un conjunto de principios, que forman un sistema íntimamente relacionado y entre los que se pueden señalar los siguientes:

a) El principio del carácter masivo y con equidad de la educación

La educación como un derecho y deber de todos es una realidad en Cuba. Ello significa las posibilidades reales de educación sin distinción de edades, sexo, raza y religión o lugar de residencia. Presupone el deber de preparar a todos para la vida en un sentido amplio.

Se ha alcanzado, de forma masiva, con equidad y calidad, el objetivo de la universalización de la enseñanza, inicialmente de la escuela primaria y posteriormente de la secundaria básica, correspondiente al nivel medio, así como el establecimiento de un sistema que abarca todos los tipos y niveles de educación para los niños, jóvenes y adultos, incluyendo a aquellos con limitaciones físicas o mentales.

\section{b) El principio de estudio y trabajo}

En el sistema educacional cubano la combinación del estudio con el trabajo -variante fundamental del principio de vincular la teoría con la práctica, la escuela con la vida y la enseñanza con la producción-, tiene profundas raíces en las concepciones sobre educación de José Martí.

Martí, como sus predecesores, no sólo defiende la enseñanza científica, sino proclama la necesidad para la América Latina, de una enseñanza científica que se sustente -como punto de partida- en la problemática económica de países como los nuestros, cuyas riquezas y recursos son eminentemente agrícolas.

La aplicación del principio en los diferentes niveles educacionales aúna dos objetivos fundamentales para la educación, uno formativo y el otro económico.

El objetivo formativo busca desarrollar una conciencia de productor de bienes sociales; en el sistema educacional cubano la combinación del estudio y el trabajo -variante fundamental del principio de vincular la teoría con la práctica, la escuela con la vida y la enseñanza con la producción, acorde con las concepciones de José Martí sobre la educación, quien resumió y enriqueció lo más progresista del ideario pedagógico cubano, eliminando los prejuicios que se derivan de la división entre el trabajo manual y el intelectual, eliminando el intelectualismo en la enseñanza y fomentando el interés por el mundo circundante.

El objetivo económico se propone integrar a la producción y al trabajo social la capacidad de centenares de miles de escolares que, dosificando adecuadamente el tiempo de estudio regular y la participación en la producción y en las actividades culturales, estéticas, deportivas y recreativas, aporte de manera concreta a su propia subsistencia alimentaria, y a la producción de bienes materiales para la sociedad.

c) El principio de la participación democrática de toda la sociedad en las tareas de la educación del pueblo

Este principio, que reconoce a la sociedad como una gran escuela, manifiesta el carácter democrático y popular de la educación cubana, que no sólo se extiende a las diferentes zonas y regiones del país y a todas las capas de la población, sino también en el hecho trascendente de que el pueblo participa en la realización y control de la educación y en la garantía de su desarrollo exitoso.

La acción y apoyo de todas las organizaciones e instituciones sociales y no gubernamentales en la labor educativa, es una condición básica de la estrategia para garantizar los niveles alcanzados y elevar la calidad de la educación en las condiciones de período especial en tiempos de paz, que enfrenta nuestro pueblo en estos instantes. 
Esto se manifiesta particularmente en el diseño cada vez más amplio de las estrategias educativas, de su control y en la toma de decisiones, que alcanza todos los niveles de la sociedad, empezando con la familia, y de los órganos del Poder Popular, hasta la Asamblea Nacional (Parlamento).

El pueblo participa en el proceso educativo mediante el análisis, la discusión, el control, la solución y el respaldo de los planes estatales en correspondencia con el principio de que "la educación es tarea de todos".

d) El principio de la coeducación y de la escuela abierta a la diversidad.

Se reafirma la aplicación de la práctica de la coeducación en todos los tipos y niveles del Sistema Nacional de Educación, con lo cual se garantiza a la mujer y al hombre el acceso a los centros de formación en cualquiera de las especialidades y profesiones que ofrece dicho Sistema.

En él se refleja la voluntad del ingreso, permanencia y trabajo por la graduación con éxito para todos, sin ningún condicionamiento por razón de sexo, raza, religión, grupo social o capacidad.

\section{e) El enfoque de género en la Educación cubana}

El acceso de las niñas y las mujeres a la educación se considera en Cuba un derecho elemental conquistado desde hace cuatro décadas, pero además se ha constatado en diversas investigaciones que el nivel escolar de la madre actúa como una variable directamente asociada a los niveles de aprendizaje de los hijos e hijas, siendo esta una razón importante para que a la educación de la mujer se le preste una atención especial.

El enfoque de género, se incluye en los programas curriculares y de forma gradual en los libros de texto, en este caso con mayor lentitud debido a las dificultades existentes con los insumos para las nuevas ediciones. A pesar de esas limitaciones, se ha incrementado en los programas de superación, capacitación y pos grado.

Para ello el Ministerio de Educación elaboró un convenio conjunto con la organización Federación de Mujeres Cubanas, donde se promueven diferentes acciones, entre ellas la investigación y la promoción en las escuelas del enfoque de género, desde el diseño de una educación no-sexista que llegue hasta el modo de vida de la escuela y el sistema de actividades y relaciones que paulatinamente contribuyen a socializar y conformar la subjetividad de los y las estudiantes.

f) El principio de la atención diferenciada y la integración escolar.
La Educación cubana trabaja, a partir de un diagnóstico profundo del niño o niña, de su familia y entorno y de cómo interactúa con él, por la atención diferenciada a los escolares, de acuerdo con sus necesidades y posibilidades, tomando en consideración las ayudas y estimulaciones oportunas que cada cual requiere, promoviendo siempre su máximo desarrollo y nunca la exclusión o la segregación en la atención educativa.

En la escuela, que es el centro más importante de la comunidad, se produce la acción de las diferentes agencias educativas, que velan por atenuar los efectos sociales ajenos al fin de la Educación y promover las condiciones educativas más favorables para el pleno desarrollo de cada individuo, en su contexto y realidades.

Cada vez más, en la escuela cubana, se promueve el tratamiento a los niños con necesidades educativas especiales; en el caso particular de aquellos con discapacidades, se trabaja de manera que estos se eduquen en el contexto natural de la escuela primaria y que sólo estén en instituciones educativas especializadas por el periodo de tiempo requerido para su integración a otro centro común.

Especial atención se brinda en todos los niveles del gobierno al fortalecimiento del trabajo encaminado a lograr la integración amplia de todos los ciudadanos sin excepción a la vida social y a las posibilidades de trabajo.

\section{g) El principio de la gratuidad}

La enseñanza es gratuita en todos los niveles. El Estado mantiene un amplio sistema de becas para todos los estudiantes y proporciona múltiples prioridades de estudio a los trabajadores a fin de alcanzar la universalización de la enseñanza².

Actualmente Cuba está enfrascada en la búsqueda de un sistema educacional que se corresponda cada vez más con la igualdad, la justicia plena, la autoestima y las necesidades morales y sociales de los ciudadanos en el modelo de sociedad que el pueblo se ha propuesto crear.

El trabajo educativo se realiza de manera más diferenciada, estable y sistemático, favorecido por el fortalecimiento que tiene lugar en la comunicación con la familia, el vínculo hogar-escuela y las relaciones afectivas que se establecen entre el profesor y los alumnos.

Se profundiza el compromiso que contraen estos profesores con su profesión de educador, es decir, el compromiso con la educación de sus alumnos.

El Profesor General Integral, por la naturaleza de su perfil profesional, logra colocar en un primer plano la

2 - http://www.rimed.cu/generales/ensenanzas.asp consultada en diciembre 7 del 2008. 
labor educativa, articulándola armónicamente con la instructiva, por ser responsable de la dirección del proceso enseñanza-aprendizaje de todas las asignaturas del currículo y no de una en particular. Logra un diagnóstico integral y sistemático de sus alumnos.

El nivel de independencia que logran los alumnos en la realización de los diferentes tipos de ejercicios evaluativos, lo que permite conocer el cumplimiento real de los objetivos de las diferentes asignaturas y del grado, por cada uno de los alumnos.

Permite un mayor control al proceso pedagógico y una evaluación más integradora del cumplimiento de los objetivos formativos.

Propicia una mayor cultura general e integral en los protagonistas del proceso pedagógico, al mismo tiempo que favorece la motivación e interés hacia el estudio y el aprendizaje.

Estos nuevos y novedosos enfoques en la vinculación temprana de la teoría y la práctica en la formación de docentes, constituyen una necesidad de la educación cubana contemporánea y responde, además, al desarrollo socio-cultural y científico-técnico alcanza-do por la humanidad. Sus fundamentos tienen raíces en las ideas pedagógicas de destacadas figuras de nuestra historia, como Félix Varela, Luz y Caballero y José Martí que tiene su continuidad histórica en la prédica constante de Fidel dirigida a la introducción de cambios y nuevas ideas en la política educacional que nos permitan una elevación continua de la calidad del proceso formativo e instructivo de las nuevas generaciones, sin abandonar la masividad y la lucha por alcanzar la plena igualdad de oportunidades $y$ posibilidades para todos.

\section{Características Curriculares}

Tensión entre lo oficial y lo privado: No existe, por cuanto no hay educación privada.

La educación en Cuba es obligatoria hasta el noveno grado de la enseñanza secundaria y gratuita en todos los niveles, incluyendo la superior. - poner la enseñanza media y superior al alcance de todos los jóvenes, al crear cientos de miles de becas.

El país cuenta con una red de 9029 escuelas primarias distribuidas por todo el territorio nacional, de ellas, 2336 en el sector urbano y 6693 en el rural, por la gran dispersión de su población. Existen 4729 multígrados con sus diferentes complejidades, lo que permite la atención de toda la matrícula de este sector, independientemente de la zona en que esté enclavada la escuela.

La tensión entre tradición y la innovación se puede evidenciar mirando retrospectivamente lo que ha sido la educación y comparando con la prospectiva, por ejemplo la generalización del uso de la televisión y el video como medios audiovisuales de gran impacto en la enseñanza primaria y media. En la actualidad se dispone de un televisor por aula en todo el país: 81 mil 169 , y un video por cada 100 alumnos. El pasado curso fueron introducidos 44 mil 790 equipos de computación y formados más de 12 mil jóvenes profesores para impartir esta enseñanza no sólo en los centros universitarios, sino también desde el preescolar. Cuba cuenta con algo más que un Canal Educativo. Transmite diariamente entre 10 y 12 horas de material educativo por los dos canales nacionales, y adicionalmente utiliza el Canal Educativo, en pleno desarrollo. Este abarca ya la Capital de la República y gran parte de las provincias Habana y Santiago de Cuba. Alcanzará a todas las capitales de provincias y ciudades mayores del país, así como a gran parte de las áreas rurales antes de finalizar el presente curso escolar. Contará con muchas más horas de transmisión escolar como parte de un sistema vinculado orgánicamente a todos los niveles y tipos de enseñanza del país.

La tensión entre el campo de producción de conocimiento y el de recontextualización pedagógica en Cuba se reduce mediante la combinación del estudio con el trabajo. Es una variante fundamental del principio de vincular la teoría con la práctica, la escuela con la vida y la enseñanza con la producción, El conocimiento como verdad absoluta y terminada no aplica porque en el fragor de la producción se enriquece con las realidades y conflictos que allí surgen. Son verdades del pueblo y para el pueblo, siguiendo las profundas raíces en las concepciones del Héroe Nacional, José Martí, sobre la educación. En este elemento tiende a disminuir la tensión entre el trabajo individual enciclopédico y el trabajo colectivo y colegiado por cuanto en el proceso de producción se producen diálogo y discusiones en forma más intensiva y permanente.

En Cuba el currículo es de reconstrucción social y, por lo tanto, tiene una intencionalidad emancipadora, lo cual alivia la tensión con las Intencionalidades generadas por el Currículo tradicional que intenta someter, ajustar las mayorías a los esquemas del estatus quo que a una redención de lo social.

Tensión entre pertenencia social y pertenencia académica se presenta al igual que en Colombia, por cuanto no hay flexibilidad y no existe diversidad curricular acorde a cada región, se observa los mismos currículos, dictados desde el centro político: La Habana, los cuales tienden a homogenizar la cultura, mediante programas nacionales. Lo anterior es reconocido por los mismos cubanos al proponer, para vigencias futuras, programas más flexibles que se ajusten a los desarrollos regionales (Ministerio de Educación República de Cuba, 2001).

No existe tensión entre los alumnos egresados de las facultades de educación y las normales (docentes en ejercicio) y los que quieren ser profesionales de la educación (los mismos docentes) por cuanto la nueva normatividad exige que pasen de ser apéndices del currículo a constructores de currículo. La práctica del 
profesor o la profesionalidad del docente, consiste, como se ha visto, en un acompañamiento del aprendizaje muy personalizado, de tal suerte que el docente es en realidad un profesional de la educación, con voluntad y vocación.

En Cuba se empezó con el proceso de Deconstrucción Curricular en cuanto que cada profesor desarrollo aptitud de organizarlo, se reúnen todo el tiempo para hablar sobre cómo mejorar el currículo, de tal manera que las prácticas culturales van ajustándose cada vez más a lo que se dice en el currículo escrito. Estas prácticas invaden en forma activa todos los campos del currículo mediante el permanente dialogo entre profesores y entre profesores y estudiantes en un seguimiento continuo. De esta forma se cierran las fisuras y grietas que existen entre la normatividad, el currículo escrito y el currículo que practican o realizan los docentes. Por otra parte el objetivo institucional cada vez es más centrado en sus propósitos de ofrecer una educación al servicio del pueblo cubano.

La educación, concebida como acompañamiento, produce condiciones básicas para la formación de profesionales que superen los procesos de transmisión y encuentren institucionalmente su identidad profesional, acorde a las nuevas exigencias y haciendo coincidir el currículo oculto con el escrito y expresado en normas, produciéndose una Deconstrucción Curricular a medida que se implementa y desarrolla el mismo, logrando calidad y no como sinónimo de cumplimiento de requisitos (Lopéz, 2001).

Independientemente de la influencia de los aspectos anteriores sobre la educación y formación de los escolares se refuerzan en el currículo, como ejes transversales: las normas de comportamiento social y ciudadana, la educación internacionalista, la solidaridad, la educación para la paz y la tolerancia, los derechos humanos, la educación ambiental, en particular, la protección del medio ambiente, la educación para la salud, el desarrollo de una sexualidad responsable, los problemas de la educación vial; entre otros.

La educación para la formación de valores humanos y universales, unidos a la educación patriótica y ciudadana, constituye sin duda, el paradigma en torno al cual se articulan el resto de los ejes transversales.

Los lineamientos para fortalecer la formación de valores y cualidades de la personalidad, encuentran su materialización en las propias potencialidades que ofrece el contenido de las asignaturas, lo que se complementa con las llamadas cátedras de valores y los turnos de reflexión y debate.

Las cátedras de formación de valores, integran como sistema el conjunto de acciones curriculares y extracurriculares que en cada centro se desarrollan para la formación de valores. En estas no solo se incorporan los profesores de mayor prestigio en la escuela, sino también alumnas y alumnos y trabajadores de la comunidad, personalidades de la cultura, la ciencia, delegados de los órganos locales de gobierno, jubilados, entre otros.

Es en la familia donde el niño aprende por primera vez a amar y cuidar la naturaleza o a destruirla y rechazarla. A la escuela le corresponde reforzar los patrones positivos que han adquirido en la familia y a eliminar aquellos que resulten negativos. Pero no solo se trata de desarrollar capacidades de orden intelectual, sino fundamentalmente que desarrollen sentimientos de amor y respeto, actitudes relacionadas con la valoración y el interés por el medio ambiente, y participen en forma activa en su mejoramiento y conservación.

Se requiere el conocimiento de la comunidad donde está radicada la escuela, desde el punto de vista laboral, así como de las familias cuyos hijos están en la escuela como un caudal inagotable de experiencias que pueden ser trasmitidas por diferentes vías a los alumnos y que elevarán su aprecio por los trabajadores y su admiración por aquellos que realizan labores en condiciones de abnegación y sacrificio.

\section{De cara al futuro:}

Cuba atraviesa por una situación económica adversa, que se ha denominado período especial, derivado fundamentalmente de los efectos del bloqueo impuesto por el Gobierno de Estados Unidos, durante más de cinco décadas.

A pesar de ello la educación conserva su vitalidad y en un futuro se piensa en facilitar la flexibilidad en educación en las distintas regiones del país, para que los planes se ajusten a la realidad cambiante y a los intereses de los educados.

Se hará un esfuerzo para que el monto de los recursos destinados a la educación corresponda con la calidad de los resultados del aprendizaje y la formación de los estudiantes. Se debe disminuir la tendencia a la teorización en la formación de maestros y profesores, con mayor vinculación de la teoría con la práctica.

\section{En Educación Superior se reforzará la tendencia a:}

- Incrementar la integración de las Universidades con las unidades productivas y de servicio.

Aumentar el grado de descentralización que posibilite que las autoridades a nivel de Universidad, facultad y departamento docente, tengan mayor posibilidad en la transformación del plan de estudio, que sin afectar los conceptos más estratégicos del mismo, lo adecuen a las necesidades y condiciones de la región y la comunidad.

El integrar en una mayor medida lo académico, lo laboral y lo investigativo, que convierta a la ciencia en la lógica fundamental del aprendizaje. 


\section{Confrontación o diálogo con la realidad colombiana}

En Colombia existe la ley 30 para las universidades y la ley 115 como ley general que rige toda la educación en Colombia. Definición de educación formal. Se entiende por educación formal aquella que se imparte en establecimientos educativos aprobados, en una secuencia regular de ciclos lectivos, con sujeción a pautas curriculares progresivas, y conducente a grados y títulos.

La educación preescolar corresponde a la ofrecida al niño para su desarrollo integral en los aspectos biológico, cognoscitivo, sicomotriz, socio-afectivo y espiritual, a través de experiencias de socialización pedagógicas y recreativas.

Currículo, según el ARTíCULO 76 Ley 115, es el conjunto de criterios, planes de estudio, programas, metodologías, y procesos que contribuyen a la formación integral y a la construcción de la identidad cultural nacional, regional y local, incluyendo también los recursos humanos, académicos y físicos para poner en práctica las políticas y llevar a cabo el proyecto educativo institucional.

La ley 115, por primera vez, propone la posibilidad al docente de ser constructor de currículo en el momento de la formulación de PEI. Esto da la posibilidad de que el docente se convierta en un investigador de currículo, adecuado a las necesidades regionales y con influencia en los planes decenales de educación, los cuales deberían ser elaborados desde las regiones, con planes regionales, cuyo conjunto conforman el plan nacional decenal de educación.

La educación se concibe como un servicio público que puede ser prestado por entes privados y oficiales. Los oficiales están obligados a impartir instrucción gratuita.

En Colombia, en palabras de Nelson E López (2001) no es lo mismo democratizar la educación que democratizar la calidad. La tensión entre lo privado y lo oficial se caracteriza por la propuesta de financiar la educación en entidades privadas con recursos estatales; caso universidad cooperativa, créditos a estudiantes para universidades privadas, becas oficiales en colegios privados, etc. En general no se democratiza la calidad por cuanto las universidades oficiales cada vez reciben menos apoyo estatal y son obligadas al autofinanciamiento mediante indicadores de calidad formulados desde perspectivas de eficiencia en peso/cantidad de alumnos atendidos.

En Colombia existe tensión entre tradición y la innovación, pues se refuerzan las carreras de siempre y no se pone énfasis en las nuevas carreras basadas en las innovaciones tanto en la virtual como en las nuevas tecnologías que ofrecen oportunidades muy novedosas en torno a carreras basadas puramente en información, materiales nuevos, tendencias conservacionistas de desarrollo sostenible, etc. Siempre salen los alumnos con ideas antiguas sobre la infinitud de los recursos de la tierra.

También existe tensión entre verdad absoluta (producida en el extranjero) y la inversión colombiana en investigación, la cual poco o nada garantiza producción de conocimiento en el país, que soporte nuestro desarrollo y no el de otras latitudes. Colombia se encuentra en el trópico y no tenemos centros de gran importancia en investigación solar. Cuando sale el sol, debido a nuestro desconocimiento en energía solar, renegamos del calor que produce, pero no agradecemos la riqueza contenida en sus rayos en cuanto a posibilidades de aprovechamiento de su gran potencial energético. En cambio, el escaso presupuesto de investigación se invierte en, por ejemplo, grupos de investigación en partículas elementales, a pesar de que su contribución al desarrollo es tan poco probable en el corto plazo y de que la investigación práctica en esos campos es tan exorbitante que los países europeos se debieron unir para poder costearla.

La intencionalidad no es emancipadora, es la de favorecer las clases poseedoras y no la desarrollar las inmensas mayorías desposeídas. La educación ni siquiera, en contra de todas las constituciones colombianas, ha sido una hija reconocida por el estado, pues siempre busca que la financien los organismos regionales: departamentos y municipios.

Se refuerza la Tensión entre el trabajo individual enciclopédico en contraposición al trabajo colectivo y colegiado. Se alimentan intereses personales en la educación y no se da importancia al trabajo colectivo, que muy poco se favorece en nuestro currículo. El único hecho colectivo de los trabajos en grupo es el de grapar o poner ganchos a cada una de las partes desarrolladas por los integrantes al momento de entregar el trabajo.

También existe Tensión entre pertenencia social y pertenencia académica pues hay muy poca flexibilidad y diversidad curricular y se trata de homogenizar todos los currículos, sin tener en cuenta las diversas realidades regionales de pie de monte, altiplano, selva, litorales y la compleja diversidad de nuestras regiones, aunada a la diversidad cultural que se ha desarrollado en esas diferentes geografías.

Se presenta Tensión entre los alumnos de los institutos superiores y medios para la formación de profesores y los que realmente quieren ser profesionales de la educación. La mayoría de los profesores no hacen de su práctica una oportunidad de investigación y mejoramiento curricular, sino que son simples apéndices de un currículo diseñado por empresas, incluso transnacionales.

Por otra parte, la educación colombiana nos exige, la deconstrucción curricular, es decir, cambiar la aptitud de organizar, de entender intervención activa que ha ido invadiendo el ámbito educativo, Nos exige ver 
fisuras y grietas, que permanecen en el currículo oculto: una cosa es lo que profesores escriben en los preparadores de clase o admiten que se debe hacer en los foros educativos y otra cosa es la que se hacen en las clases, con sus alumnos. La objetividad institucional esta descentrada en la medida que no ofrece condiciones básicas para la formación de profesionales que superen los procesos de transmisión de conocimiento y no están diseñadas para el desarrollo de una identidad profesional del docente colombiano, adecuado a las realidades regionales.

Los docentes siempre interpretan la acreditación con equivalente de acatamiento de requisitos legales, en lugar de ser un compromiso de acompañamiento de sus estudiantes para la obtención de sus logros.

Es imperante que se devele la estructura conceptual del currículo oculto, La herencia no pensada (Lopéz, 2001) con la que los docentes nos enfrentamos a la tarea de enseñar.

\section{Comentario Final}

En Cuba, después de la revolución, la enseñanza debió ser cambiada, pues fue concebida para élites minoristas, y no se ajustaba a los requerimientos de una Educación para la totalidad de los niños y adolescentes. Era necesario garantizar la interdisciplinariedad y la transdisciplinariedad, las cuales constituyen la gramática de un nuevo paradigma formativo, que implique una variación y modificación sustantiva de las estructuras de poder y control que determinan la dinámica educativa de la educación formal en todos sus niveles (López, 2002). Estas circunstancias garantizan la continuidad de la revolución, expresando en sus formas de sentir, de pensar y de actuar en forma integral, permitiendo a los adolescentes conocer y entender su pasado, enfrentar su vida presente y su preparación futura, adoptando conscientemente una opción de vida socialista, que garantice la continuidad de la revolución.

En Colombia se tiene la percepción de que todo se resuelva emitiendo decretos. Hay una legislación abundante y pocos resultados. En Cuba, no hay ley general de educación, sólo el mandato constitucional, pero abundantes logros. En Colombia se pierde mucho tiempo capacitando a maestros en nuevas normas, que rápidamente se cambian sin evaluación, sin previo aviso. Lo anterior implica grandes impactos negativos para los profesores que apenas estaban dando el primer paso para el cambio, cuando ya deben volver a capacitarse para el siguiente capricho del MEN.
En Cuba se hace un seguimiento desde la tierna infancia, incluso desde antes de nacer, educando al padre para que él inicie la educación. Lo integra desde ese momento a la educación. En Colombia se envía al niño lleno de tareas a la casa y los padres, sin ninguna preparación, torpedean el proceso educativo, sometiéndolo a injustas presiones y reclamaciones.

La ley 115, no establece como objetivo de la educación preescolar desarrollar habilidades comunicativas en lectura y escritura (artículo 16). Este es un objetivo de la educación básica primaria (artículo 20), pero las editoriales lo han impuesto así. De esta forma, las editoriales son las que, en últimas, están rigiendo la educación, por cuanto los maestros muy dócilmente siguen estas cartillas.

\section{Referencias Bibliográficas}

Banco de la República, 2014. PIB. Metodología año base 2005. Recuperado de http://www.banrep.gov.co/es/pib?__utma= 1.59111582.1402515500.1402515500.1402515500.1\&_utmb=1. $1.10 .1402515500 \&$ \&_utmc $=1 \&_{\text {_-_utm }}=-\&_{\text {____utmz }}=1.1402515500$. 1.1.utmcsr=google|utmccn $=$ (organic) $\mathbf{u t m c m d}=$ organic|utmctr $=(\mathbf{n}$ ot\%20provided)\&_utmv=-\&_utmk=110769669

Fundación Compartir, 2014. Tras la excelencia docente: Cómo mejorar la calidad de la educación para todos los colombianos. Recuperado de http://www.fundacioncompartir.org/pdf/ Resumen_Ejecutivo.pdf

Icfes, 2014. PISA. Recuperado de http://www.icfes.gov.co/ investigacion/evaluaciones-internacionales/pisa

López, N. E. 2001. La deconstrucción curricular. Bogotá. Cooperativa ed magisterio.

López, N. E. 2002. Los proyectos educativos institucionales renovadores, PEIR. Rev. Paideia N10. Agosto-diciembre 2002. Universidad Surcolombiana... ISSN-012-0308. Neiva.

MEN, 2008. Colombia: qué y cómo mejorar a partir de la prueba PISA. Recuperado de

http://www.mineducacion.gov.co/1621/article-162392.html

MEN, 2005. Formación y desarrollo profesional docente. Recuperado de http://www.mineducacion.gov.co/1621/propertyvalue31598.html

MEN, 2009. Cuba Un sistema de mejoramiento continuo. Recuperado de http://www.mineducacion.gov.co/1621/article-89947.html

Ministerio de Educación República de Cuba, 2001. Contenido de la educación y estrategias de aprendizaje para el siglo XXI. Informe presentado al Seminario Subregional sobre el Desarrollo Curricular para Aprender a Vivir Juntos. La Habana, Cuba, 15 al 18 de mayo del 2001.

Presidencia de Colombia, 2013. Este es el presupuesto de la nación para 2014. Recuperado de http://www.urnadecristal.gov.co/ gestion-gobierno/presupuesto-colombia-2014-sena-agro

Revista Semana, 2014. Fundación compartir propone ruta para mejorar educación en el país. Recuperado de http://www.semana. com/nacion/articulo/fundacion-compartir-propone-ruta-paramejorar-educacion-en-el-pais/377284-3.\%20consultado\%2015\% 20de\% 20febrero\%202014

Sistema Educativo Nacional de Cuba, 1995. Ministerio de Educación de Cuba y Organización de Estados Iberoamericanos; [informe realizado por Miguel Varela Hernández... (et. al.)].- La Habana, Cuba, 1995

Unesco, 2006. Evaluación del desempeño y carrera profesional docente. Recuperado de http://unesdoc.unesco.org/images/0015 /001529/152934s.pdf 